\title{
Evaluación de competencias genéricas y socioemocionales por parte de universitarios
}

\author{
José Antonio Morales Rodríguez ${ }^{1}$ y Francisco Manuel Morales Rodríguez ${ }^{2}$ \\ ${ }^{1}$ Alicante (España); ${ }^{2}$ Universidad de Granada (España)
}

En España el sistema educativo ha apostado de manera explícita por un aprendizaje basado en competencias, tal y como queda reflejado en la Ley Orgánica 8/2013 para la mejora de la calidad educativa, en la que se afirma que la mezcla de competencias y conocimientos diversos es la fortaleza para una sociedad más abierta, global y participativa que exige colaboración y trabajo en equipo. Es necesario, como se hace en el presente trabajo, aplicar un instrumento que permita optimizar la evaluación de competencias genéricas en una muestra de estudiantes universitarios, así como contrastar la importancia que dan a las mismas para su desempeño laboral personas con una exitosa trayectoria o desempeño en el ámbito empresarial. En este trabajo se dan a conocer los resultados de aplicar un instrumento para la evaluación de competencias transversales a universitarios consideradas importantes tanto por los propios universitarios como por personas de éxito en el ámbito empresarial, los dos principales protagonistas para la adquisición o desarrollo de competencias transversales que se demandan desde el Espacio Europeo de Educación Superior. Los datos obtenidos se consideran relevantes para contribuir a que el estudiantado pueda desarrollar las habilidades genéricas y competencias socioemocionales que los hagan atractivos para los empleadores y aptos para el mercado laboral.

Palabras clave: Evaluación, competencias genéricas, estudiantes universitarios.

Assessment of generic and socioemotional competences by university students. The Spanish education system has opted explicitly for competency-based learning, as set out in Organic Law $8 / 2013$ for the improvement of educational quality, which states that a mixture of diverse competences and knowledge provides the backbone for a more open, broader and participative society requiring collaboration and team work. In light of the above, the present work describes a much-needed instrument that: (i) optimises the assessment of generic competences in a sample of university students, and (ii) determines the importance that people with a successful career place on these competences with respect to their performance in the workplace or business environment. We present the results of applying such an instrument to the assessment of transversal skills considered relevant by both university students and graduates who have achieved success in the business world, which correspond to the two primary groups involved in the acquisition and development of transversal skills demanded by the European Higher Education Area. The data obtained in this study are considered relevant as they allow students to develop the generic skills and socioemotional competences that will make them attractive to future employers and suitably prepared for the labour market.

Keywords: Assessment, generic competences, university students.

Correspondencia: Francisco Manuel Morales Rodríguez. Departamento de Psicología Evolutiva y de la Educación. Universidad de Granada. Campus Universitario de La Cartuja. C.P.: 18071. Granada (España). E-mail: fmmorales@ugr.es 
El proyecto Definición y Selección de las Competencias (DeSeCo) de la OCDE indica que la competencia va más allá de la yuxtaposición de conocimiento y habilidades, involucrando la capacidad para cumplir con demandas complejas, para lo cual clasifica tales competencias en tres grupos: a) uso de herramientas interactivamente (lenguaje, tecnología; b) interactuar en grupos homogéneos; y c) actuar de forma autónoma (Organisation for Economic Cooperation and Development (OECD), 2005).

En España el sistema educativo ha apostado de manera explícita por un aprendizaje basado en competencias, tal y como queda reflejado en la Ley Orgánica 8/2013 para la mejora de la calidad educativa, en la que se afirma que la mezcla de competencias y conocimientos diversos es la fortaleza para una sociedad más abierta, global y participativa que exige colaboración y trabajo en equipo.

Las competencias pueden clasificarse en dos tipos: específicas y genéricas o transversales. Las competencias específicas son las correspondientes a un determinado ámbito de conocimiento o actividad, mientras que las competencias genéricas se refieren a aquellas que son comunes a todos los ámbitos de conocimiento u ocupación (Clemente-Ricolfe y Escribá-Pérez, 2013).

Las definiciones de competencias genéricas son variadas. En principio, las habilidades genéricas son las habilidades que los estudiantes necesitan para convertirse en personas más capacitadas a nivel transversal en su campo de estudio, trabajo y otros aspectos de su vida y son un aspecto importante y reconocido de la educación universitaria (Allan y Clarke, 2007).

Las definiciones de competencias genéricas son variadas. En el ámbito del Proyecto Tuning las competencias genéricas se agrupan en tres clases (Tuning Educational Structures in Europe, 2008): a) instrumentales; b) interpersonales; y c) sistémicas. Es un hecho generalmente reconocido que es necesario seguir mejorando la capacitación de los recién titulados en enseñanzas superiores para implementar en el mundo laboral las habilidades comprendidas en el ámbito de las competencias genéricas (Badcock, Pattison, y Harris, 2010). A partir de estos tres tipos de competencias genéricas se genera una lista de 30 competencias básicas de carácter genérico entre la que se incluyen la planificación y gestión del tiempo, comunicación oral y escrita, capacidad de aprender, capacidad crítica y autocrítica, de adaptación a nuevas situaciones, creatividad, resolución de problemas, habilidades interpersonales, trabajo autónomo, etc. No obstante, no existe una definición universalmente reconocida ni un listado de competencias genéricas aceptado por todos (Ho, Luk, y Chan, 2014).

La experiencia industrial demuestra que las habilidades genéricas son importantes ya que contribuyen al aumento de la eficiencia y la productividad del trabajo, y son recursos intangibles valiosos para lograr una ventaja competitiva. Para los estudiantes que se gradúan, las habilidades genéricas aumentan sus posibilidades de empleabilidad, el proceso por el cual pueden conseguir un trabajo en concordancia con su 
educación, actitud y motivación (Hande, Mohammed, y Komatil, 2015; Vainikainen, Hautamaki, Hotulainen, y Kupiainen, 2015). Por esta razón, muchos autores identifican habilidades genéricas con habilidades de empleabilidad, que se pueden definir como "habilidades requeridas no solo para obtener empleo sino también para progresar dentro de una empresa para alcanzar el potencial y contribuir con éxito a las direcciones estratégicas de la empresa" (Curtin, 2004, p. 39).

Por todo lo anterior, es necesario, como se hace en el presente trabajo, aplicar un instrumento que permita optimizar la evaluación de competencias genéricas en una muestra de estudiantes universitarios, así como contrastar la importancia que dan a las mismas para su desempeño laboral personas con una exitosa trayectoria o desempeño en el ámbito empresarial.

\section{MÉTODO}

\section{Participantes}

Los participantes fueron 45 estudiantes universitarios pertenecientes a la Facultad de Ciencias de la Educación. La muestra está equilibrada según el sexo. También se entrevistó a algunas personas con puestos importantes en el ámbito empresarial sobre la importancia que otorga, en términos generales, y de forma cualitativa a estas competencias.

\section{Instrumentos}

Para la evaluación de las competencias genéricas se ha aplicado el Cuestionario para la evaluación de competencias genéricas en estudiantes universitarios elaborado por Solanes, Núñez, y Rodríguez-Marín (2008, 2012). Se trata de un cuestionario que permite evaluar competencias instrumentales (desempeño del trabajo, habilidades para la gestión); competencias sistémicas (capacidad de aprendizaje, liderazgo y motivación para el trabajo) así como las llamadas competencias interpersonales como las relacionadas con las llamadas relaciones interpersonales y trabajo en equipo. Está formado por un total de 45 ítem con formato de respuesta en seis escalones: 1. Siempre; 2. Muchísimas veces; 3. Con frecuencia; 4. Ordinariamente; 5. Ocasionalmente; y 6. Casi nunca. Concretamente lo forman 12 ítems para desempeño del trabajo; 7 ítems para habilidades para la gestión; 10 para liderazgo; 7 motivación para el trabajo; 4 para capacidad de aprendizaje; y 5 ítems para la categoría de relaciones interpersonales y trabajo en equipo. Este instrumento se viene utilizando en distintos estudios sobre competencias genéricas (por ejemplo, se aplicó en el estudio de Agudo, Hernández-Linares, Rico, y Sánchez, 2013). 


\section{Procedimiento}

En una de las prácticas sobre Psicología de la Educación tras responder al cuestionario para que el alumnado se autoevalúe y proponer la lectura del artículo sobre competencias de este cuestionario se reflexionaron y se generó un debate común a la clase sobre la importancia de las competencias genéricas y se mostró un instrumento sobre competencias que, de forma totalmente voluntaria, anónima y garantizando la confidencialidad de la información y el tratamiento global de los datos cumplimentaron.

\section{RESULTADOS}

A continuación, en la tabla 1 se muestran las puntuaciones medias obtenidas por esta muestra de estudiantes en cada una de las competencias genéricas evaluadas.

Tabla 1. Medias en competencias genéricas en la muestra de universitarios

\begin{tabular}{llcc}
\hline \multicolumn{1}{c}{ Competencias } & Media & Desviación Típica \\
\hline 1. & Competencias instrumentales & & \\
\hline 1.1. & Desempeño del trabajo & 31.66 & 6.65 \\
\hline 1.2. & Habilidades para la gestión & 16.08 & 5.16 \\
\hline 2. & Competencias sistémicas & & \\
\hline 2.1. & Liderazgo & 26.18 & 4.89 \\
\hline 2.2. & Motivación para el trabajo & 16.77 & 3.22 \\
\hline 2.3. & Capacidad de aprendizaje & 9.91 & 3.30 \\
\hline 3. & Competencias interpersonales & \\
\hline 3.1. & Relaciones interpersonales y trabajo en equipo & 36.61 & 7.92 \\
\hline
\end{tabular}

Del debate que se ha generado y las entrevistas con empleadores puede comprobarse que entre las competencias que hay que seguir fomentando se encuentran, por ejemplo, aquellas que puedan relacionarse con una adecuada gestión del tiemplo y capacidad de planificación para afrontar las tareas. Asimismo, se considera que es cada vez más necesario fomentar competencias como que pueden relacionarse con una comunicación interpersonal eficaz y adecuadas habilidades sociolaborales, etc.

A continuación, en la tabla 2 se presenta las puntuaciones medias y desviaciones típicas en cada ítem del cuestionario elaborado por Solanes et al. (2008) que resultó muy útil para que el alumnado se autoevalúe y debatir desde un punto de vista educativo sobre estas cuestiones. 
MORALES y MORALES. Evaluación de competencias

Tabla 2. Medias obtenidas en cada ítem del cuestionario de competencias genéricas aplicado

\begin{tabular}{|c|c|c|c|}
\hline \multicolumn{2}{|c|}{ Ítem del cuestionario } & \multirow{2}{*}{$\frac{\text { Media }}{2.46}$} & \multirow{2}{*}{$\begin{array}{c}\text { Desviación típica } \\
.87\end{array}$} \\
\hline 1. & $\begin{array}{l}\text { Conocimientos de la } \\
\text { carrera }\end{array}$ & & \\
\hline 2. & $\begin{array}{l}\text { Capacidad de } \\
\text { concentración para el } \\
\text { estudio }\end{array}$ & 2.85 & .55 \\
\hline 3. & $\begin{array}{l}\text { Capacidad de innovación } \\
\text { en las prácticas o trabajos }\end{array}$ & 2.92 & .75 \\
\hline 4. & $\begin{array}{l}\text { Valoración de resultados } \\
\text { académicos }\end{array}$ & 2.38 & 1.0 \\
\hline 5. & Capacidad de adaptación & 2.0 & 1.15 \\
\hline 6. & $\begin{array}{l}\text { Capacidad de previsión, } \\
\text { planificación }\end{array}$ & 2.38 & 1.19 \\
\hline 7. & $\begin{array}{l}\text { Organización de tu tiempo } \\
\text { de estudio y trabajo }\end{array}$ & 2.33 & 1.07 \\
\hline 8. & $\begin{array}{l}\text { Capacidad para organizar } \\
\text { equipos de trabajo }\end{array}$ & 2.15 & 1.14 \\
\hline & $\begin{array}{l}\text { Capacidad de } \\
\text { aprovechamiento óptimo } \\
\text { de los propios recursos }\end{array}$ & 2.30 & .75 \\
\hline 10. & $\begin{array}{l}\text { Capacidad de } \\
\text { aprovechamiento óptimo } \\
\text { de los recursos de la } \\
\text { Universidad }\end{array}$ & 2.46 & .51 \\
\hline 11. & Capacidad de negociación & 3.07 & 1.25 \\
\hline 12. & $\begin{array}{l}\text { Resolución de problemas } \\
\text { en materias de clase, en las } \\
\text { prácticas }\end{array}$ & 2.46 & .97 \\
\hline 13. & Capacidad de síntesis & 2.61 & .87 \\
\hline & Competitividad & 3.0 & 1.63 \\
\hline & $\begin{array}{l}\text { Mantenimiento de su } \\
\text { rendimiento habitual }\end{array}$ & 3.15 & 1.14 \\
\hline 16. & $\begin{array}{l}\text { Actúas con desenvoltura y } \\
\text { firmeza en las situaciones } \\
\text { de tensión }\end{array}$ & 2.58 & .79 \\
\hline & $\begin{array}{l}\text { Capacidad para trabajar en } \\
\text { equipo }\end{array}$ & 2.07 & .77 \\
\hline 18. & $\begin{array}{l}\text { Coordinación en las } \\
\text { prácticas o trabajos }\end{array}$ & 2.07 & .75 \\
\hline & $\begin{array}{l}\text { Voluntariedad en el trabajo } \\
\text { en equipo, en las prácticas }\end{array}$ & 2.38 & .87 \\
\hline 20. & $\begin{array}{l}\text { Facilidad para relacionarte } \\
\text { con tus compañeros }\end{array}$ & 1.61 & .50 \\
\hline 21. & Comunicación & 1.84 & .69 \\
\hline 22. & $\begin{array}{l}\text { Habilidades en las } \\
\text { relaciones interpersonales }\end{array}$ & 1.92 & .86 \\
\hline 23. & $\begin{array}{l}\text { Facilidad para relacionarte } \\
\text { con tus profesores }\end{array}$ & 2.69 & .75 \\
\hline 24. & Emprendedor & 2.53 & 1.19 \\
\hline & $\begin{array}{l}\text { Capacidad para conseguir } \\
\text { que los demás alumnos } \\
\text { acepten tus ideas y } \\
\text { propuestas }\end{array}$ & 2.53 & .66 \\
\hline & Inspiras confianza & 1.77 & .73 \\
\hline
\end{tabular}


MORALES y MORALES. Evaluación de competencias

Tabla 2(continuación). Medias obtenidas en cada ítem del cuestionario de competencias genéricas aplicado

\begin{tabular}{|c|c|c|c|}
\hline \multicolumn{2}{|c|}{ Ítem del cuestionario } & \multirow{2}{*}{$\begin{array}{l}\text { Media } \\
2.67\end{array}$} & \multirow{2}{*}{$\begin{array}{c}\text { Desviación típica } \\
.78\end{array}$} \\
\hline & $\begin{array}{l}\text { Habitualmente, persuades y } \\
\text { obtienes ventajas sin } \\
\text { provocar hostilidades }\end{array}$ & & \\
\hline 28. & $\begin{array}{l}\text { Capacidad de aceptar con } \\
\text { facilidad nuevas } \\
\text { responsabilidades o nuevos } \\
\text { cargos }\end{array}$ & 2.23 & .60 \\
\hline 29. & $\begin{array}{l}\text { Capacidad de relación con } \\
\text { los demás compañeros }\end{array}$ & 1.92 & .64 \\
\hline 30. & $\begin{array}{l}\text { Te proporciona acusada } \\
\text { satisfacción la posibilidad } \\
\text { de dirigir personas y } \\
\text { recursos }\end{array}$ & 2.67 & .98 \\
\hline 31. & $\begin{array}{l}\text { Generas buena imagen de } \\
\text { la Universidad ante el } \\
\text { exterior }\end{array}$ & 2.54 & .88 \\
\hline 32. & $\begin{array}{l}\text { Capacidad de conocer tus } \\
\text { propias características } \\
\text { personales y profesionales }\end{array}$ & 2.50 & 1.16 \\
\hline 33. & Estabilidad & 2.38 & 1.04 \\
\hline 34. & Autoconfianza & 2.46 & 1.13 \\
\hline 35. & Motivación & 2.54 & .97 \\
\hline 36. & $\begin{array}{l}\text { Mantenimiento del } \\
\text { rendimiento habitual }\end{array}$ & 2.31 & 1.03 \\
\hline 37. & $\begin{array}{l}\text { Capacidad para actuar con } \\
\text { desenvoltura }\end{array}$ & 2.16 & .72 \\
\hline 38. & $\begin{array}{l}\text { Te atrae fuertemente } \\
\text { conseguir los objetivos } \\
\text { marcados }\end{array}$ & 2.08 & .76 \\
\hline 39. & $\begin{array}{l}\text { Aceptas con facilidad } \\
\text { nuevas responsabilidades }\end{array}$ & 2.38 & .77 \\
\hline & $\begin{array}{l}\text { Resultados de los } \\
\text { exámenes últimos del } \\
\text { alumno/a }\end{array}$ & 2.15 & 1.28 \\
\hline 41. & $\begin{array}{l}\text { En tu trabajo habitualmente } \\
\text { encuentras soluciones } \\
\text { nuevas y originales a la vez } \\
\text { que aportas nuevas } \\
\text { perspectivas al mismo }\end{array}$ & 2.58 & .90 \\
\hline 42. & $\begin{array}{l}\text { Te adaptas bien a las } \\
\text { nuevas situaciones }\end{array}$ & 1.85 & .90 \\
\hline & $\begin{array}{l}\text { Eres capaz de establecer } \\
\text { sistemas para el } \\
\text { aprovechamiento óptimo } \\
\text { de los recursos }\end{array}$ & 2.31 & .75 \\
\hline & $\begin{array}{l}\text { Ante cualquier dificultad, } \\
\text { consigues superar tus } \\
\text { problemas sin necesidad de } \\
\text { recurrir a tus superiores }\end{array}$ & 2.58 & .90 \\
\hline 45. & $\begin{array}{l}\text { ¿Podrías desempeñar otro } \\
\text { puesto de trabajo diferente } \\
\text { del que te estás formando? }\end{array}$ & 2.75 & 1.42 \\
\hline
\end{tabular}

Las puntuaciones medias más elevadas por el grupo clase que ha cumplimentado dicho cuestionario se han obtenido en los ítems 2, 7, 15, 45. En el ítem 2 la puntuación media es de 2.5 (muchísimas veces) referido a la capacidad para 
concentrarse. En el 7 la media es de 2.33 para organización y tiempo en el estudio. Para el ítem 15 referido a "mantenimiento de su rendimiento habitual" la puntuación es de $3.15 \mathrm{y}$ para el ítem 45 referido a la se ve capaz de desempeñar otro puesto de trabajo distinto al que está recibiendo formación la puntuación media es de 2.75 .

Las puntuaciones medias inferiores se han obtenido para los ítems 20 (facilitad para relacionarte con tus compañeros) y el ítem 26 (inspiras confianza) cuyas respectivas medias son de 1.61 y 1.77 que evidencian que el alumnado autopercibe positivamente al autovalorar dichas competencias personales.

Se valora el hecho de cada vez se proponen actividades más interactivas, que fomentan el pensamiento divergente, la creatividad, el trabajo en grupo, la atención a la diversidad, la gestión de emociones y en las que se muestran ejemplos de su utilidad para la vida profesional o las implicaciones educativas que puedan derivarse de las mismas.

\section{DISCUSIÓN Y CONCLUSIONES}

En este trabajo se dan a conocer los resultados de aplicar un instrumento para la evaluación de competencias transversales tanto a universitarios como a algunas personas de éxito en el ámbito empresarial, los dos principales protagonistas para la adquisición o desarrollo de competencias transversales que se demandan desde el Espacio Europeo de Educación Superior. Los datos obtenidos se consideran relevantes para contribuir a que el estudiantado pueda desarrollar las habilidades genéricas que los hagan atractivos para los empleadores y aptos para el mercado laboral.

En el debate que se ha generado tanto por el alumnado como por parte de las personas entrevistadas se viene considerando la importancia de que en un mundo globalizado y cambiante desde el ámbito universitario se contribuya aún más al fomento de competencias transversales interpersonales como las relacionadas con el afrontamiento centrado en la gestión de emociones y estrés cotidiano ya sea laboral o académico. También se refleja que cada vez se va otorgando más importancia al trabajo en equipo como garantía de éxito académico y/o socio-laboral.

Los resultados exhiben que el alumnado de la presente muestra en lo que se refiere, por ejemplo, a competencias interpersonales consideran que inspiran confianza y son fáciles las relaciones interpersonales con el resto de los compañeros.

Con respecto a dichas competencias interpersonales como las relacionadas con la gestión emocional, se puede localizar algún estudio reciente (Martos et al., 2018) que pone de manifiesto la importancia de la inteligencia emocional para prevenir el burnout y afrontar las tareas y actividades generadoras de estrés académico en el alumnado de Ciencias de la Salud. Asimismo, otro estudio (Morales, Giménez, y Morales, 2018) encontró que la inteligencia emocional guarda relación con la autoeficacia emprendedora 
que es también una variable relevante para el desarrollo de competencias transversales como las de iniciativa y espíritu emprendedor.

Otra competencia genérica o de amplio espectro para desarrollar en el ámbito universitario es la de comunicación oral y escrita para la que es necesario considerar, entre otros aspectos, para el control de calidad de los trabajos académicos que como plantean Arias-Gundín y Fidalgo (2017) la planificación es la estrategia de escritura de más influencia y el grado de dificultad de la tarea incide en la complejidad de las estrategias que emplean los estudiantes en su proceso de escritura.

En términos generales, puede considerarse positiva la valoración que hace este grupo respecto a cómo se está contribuyendo con las actividades que se están realizando a la adquisición de las competencias genéricas desde el ámbito universitario.

En ese sentido, una fortaleza u oportunidad es el hecho de que los principales protagonistas del proceso de enseñanza/aprendizaje como son los alumnos perciben que se está contribuyendo desde el ámbito universitario con la adquisición o fomento de dichas competencias genéricas para facilitar su futura inserción socio-laboral.

Los continuos avances tecnológicos y la globalización demandan de algún modo no solo habilidades y conocimientos especializados, sino también el desarrollo de habilidades genéricas United Nations Educational, Scientific and Cultural Organization (UNESCO, 2012).

Los nuevos retos y desafíos que requieren soluciones cada vez más complejas demandas que de algún modo desde las aulas universitarias se fomente cada vez más el pensamiento crítico, el pensamiento divergente y la creatividad, y valores como los relacionados con la empatía y el trabajo a favor de otros.

Para concluir, puede señalarse la necesidad de la formación en competencias genéricas desde la Educación Superior ya que se trata de capacidades altamente demandadas por el mundo laboral, especialmente en aquellas competencias que han sido objeto de menor atención o a las que se proporciona una menor importancia para lo que se requiere de evaluaciones más precisas con instrumentos validados.

\section{REFERENCIAS}

Agudo, J.E., Hernández-Linares, M., Rico, R., y Sánchez, H. (2013). Competencias transversales: percepción de su desarrollo en el grado en ingeniería en diseño industrial y desarrollo de productos. Formación Universitaria, 6(5), 39-50. doi: 10.4067/S071850062013000500006.

Allan, J., y Clarke, K. (2007). Nurturing supportive learning environments in higher education through the teaching of study skills: embed or not to embed? International Journal of Teaching and Learning in Higher Education, 19(1), 64-76.

Arias-Gundín, O., y Fidalgo, R. (2017). El perfil escritor como variable moduladora de los procesos involucrados en la composición escrita en estudiantes universtiarios. European Journal of Investigation in Health, Psychology and Education, 7(1), 59-68. 
Badcock, P.B., Pattison, P.E., y Harris, K.L. (2010). Developing Generic Skills through University Study: A Study of Arts, Science and Engineering in Australia. Higher Education, 60(4), 441-458.

Clemente-Ricolfe, J.S., y Escribá-Pérez, C. (2013). Análisis de la percepción de las competencias genéricas adquiridas en la universidad. Revista de Educación, 362. doi: 10.4438/1988592X-RE-2013-362-241.

Curtin, P. (2004). Employability skills for the future. In J. Gibb (Ed.). Generic skills in vocational education and training. Research readings. (pp.38-68). Adelaide: National Centre for Vocational Education Research.

Hande, S., Mohammed, C.A., y Komatil, R. (2015). Acquisition of knowledge, generic skills and attitudes through problem based learning: Student perspectives in a hybrid curriculum. Journal of Taibah University Medical Sciences, 10(1), 21-25.

Ho, R.M., Luk, L.Y., y Chan, C.K. (2014). A review of literature on challenges and obstacles to implementation of generic skills. 8th International Technology, Education and Development Conference. Valencia.

Ley Orgánica 8/2013, de 9 de diciembre, para la mejora de la calidad educativa (LOMCE).

Martos, A., Pérez-Fuentes, M.C., Molero, M.M., Gázquez, J.J., Simón, M.M., y Barragán, A.B. (2018). Burnout y engagement en estudiantes de Ciencias de la Salud. European Journal of Investigation in Health, Psychology and Education, 8(1), 23-36.

Morales, F.M., Giménez, J.M., y Morales, A.M. (2018). Relaciones entre autoeficacia emprendedora y otras variables psicoeducativas en universitarios. European Journal of Investigation in Health, Psychology and Education, 8(2), 91-102.

Solanes, A., Núñez, R., y Rodríguez-Marín, J. (2008). Elaboración de un cuestionario para la evaluación de competencias genéricas en estudiantes universitarios. Apuntes de Psicología, 26(1), 35-49.

Solanes, A., Núñez, R., y Rodríguez-Marín, J. (2012). Elaboración de un cuestionario para la evaluación de competencias genéricas en estudiantes universitarios. Apuntes de Psicología, 30(1-3), 513-522.

Tuning Educational Structures in Europe. (2008). Tuning Educational Structures in Europe. Univerities' contribution to the Bologna Process. An Introduction. Bilbao: Publicaciones de la Universidad de Deusto.

United Nations Educational, Scientific and Cultural Organization (UNESCO). (2012). Transforming Technical and Vocational Education and Training Building skills for work and life. Paris: UNESCO.

Vainikainen. M.P., Hautamaki, J., Hotulainen, R., y Kupiainen, S. (2015). General and specific thinking skills and schooling: Preparing the mind to new learning. Thinking Skills and Creativity, 18, 53-64.

Recibido: 25 de junio de 2018

Recepción Modificaciones: 22 de agosto de 2018

Aceptado: 26 de octubre de 2018 